The American Journal of Chinese Medicine, Vol. 45, No. 2, 403

(C) 2017 World Scientific Publishing Company

Institute for Advanced Research in Asian Science and Medicine

DOI: $10.1142 / S 0192415 X 1792001 X$

\title{
Erratum
}

\section{The Inhibitory Effect of Abietic Acid on Melanoma Cancer Metastasis and Invasiveness In Vitro and In Vivo}

[The American Journal of Chinese Medicine, 43(8): 1697-1714, 2015]

\author{
Yih-Shou Hsieh, ${ }^{*}{ }^{\dagger}$ Shun-Fa Yang, ${ }^{\star}$ Yi-Hsien Hsieh, ${ }^{\dagger}$ Chia-Hung Hung, ${ }^{\dagger}$ \\ Shu-Chen Chu, ${ }^{\S}$ Sheng-Han Yang ${ }^{\dagger}$ and Pei-Ni Chen ${ }^{*}{ }^{*}$ \\ *Clinical Laboratory, Chung Shan Medical University Hospital, Taichung, Taiwan \\ †nstitute of Biochemistry, Microbiology and Immunology \\ Institute of Medicine, Chung Shan Medical University, Taichung, Taiwan \\ \$Institute and Department of Food Science \\ Central Taiwan University of Science and Technology, Taichung, Taiwan
}

Published 10 March 2017

We have noticed a misplaced figure, Fig. 8B, in our above-mentioned paper. The following is the corrected version of the figure.

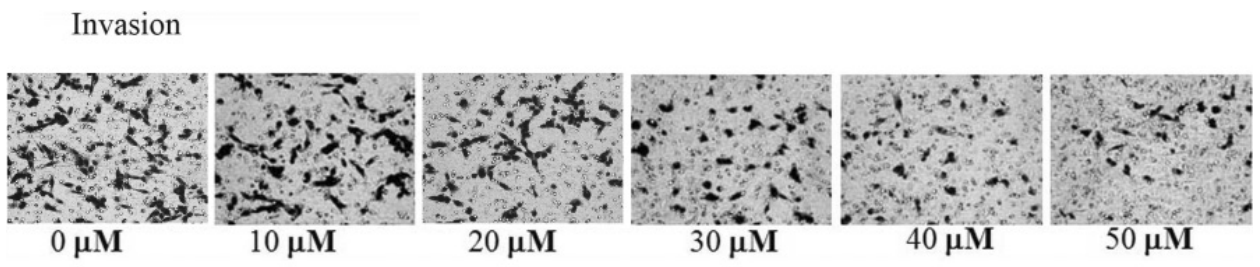

(B) 